\title{
Alterstice
}

Revue internationale de la recherche interculturelle

International Journal of Intercultural Research

Revista International de la Investigacion Intercultural

\section{Le devoir d'insoumission. Regards croisés sur l'occupation américaine d'Haïti (1915-1934), sous la direction de Roberson Edouard et de Fritz Calixte}

\section{Mélissa Arneton}

Volume 8, numéro 1, 2018

URI : https://id.erudit.org/iderudit/1052613ar

DOI : https://doi.org/10.7202/1052613ar

Aller au sommaire du numéro

Éditeur(s)

Alterstice

ISSN

1923-919X (numérique)

Découvrir la revue

Citer ce compte rendu

Arneton, M. (2018). Compte rendu de [Le devoir d'insoumission. Regards croisés sur l'occupation américaine d'Haïti (1915-1934), sous la direction de Roberson

Edouard et de Fritz Calixte]. Alterstice, 8(1), 121-124.

https://doi.org/10.7202/1052613ar d'utilisation que vous pouvez consulter en ligne. 


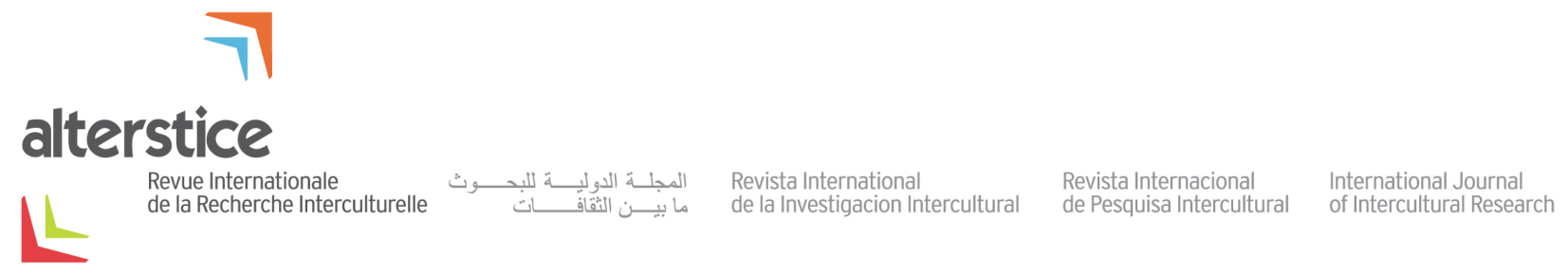

NOTE DE LECTURE

\title{
Le devoir d'insoumission. Regards croisés sur l'occupation américaine d'Haïti (1915-1934), sous la direction de Roberson Edouard et de Fritz Calixte
}

\author{
Mélissa Arneton ${ }^{1}$
}

\author{
Rattachement de l'auteure \\ ${ }^{1}$ GRHAPES / INSHEA - Université Paris-Lumière, Paris, France
}

\section{Correspondance}

melissa.arneton@inshea.fr

\section{Références de l'ouvrage}

Edouard, R. et Calixte, F. (dir.) (2016). Le devoir d'insoumission. Regards croisés sur l'occupation américaine d'Haïti (1915-1934). Québec : Presses de l'Université Laval.

\section{Pour citer cet article}

Arneton, M. (2018). Le devoir d'insoumission. Regards croisés sur l'occupation américaine d'Haïti (1915-1934), sous la direction de Roberson Edouard et de Fritz Calixte [Note de lecture]. Alterstice, 8(1), 121-124.

\section{Présentation du volume}

Cet ouvrage en sciences sociales, paru au quatrième trimestre 2016, renvoie à une page méconnue de l'histoire de la Caraïbe et qui fait l'objet de peu de travaux scientifiques : l'occupation entre 1915 et 1934 de la première république noire, Haïti, par la première république blanche, les États-Unis d’Amérique. Le collectif d'auteurs mené par Roberson Edouard et Fritz Calixte présente de manière argumentée différents points de vue sur une situation d'occupation qui, loin de se limiter à un rapport néocolonial basé sur des rapports ethnicisés, renvoie à une mobilisation culturelle et identitaire majeure. Douze chapitres, regroupés en trois parties, permettent d'aborder ce que les auteurs appellent le "devoir d'insoumission " en tant [qu'] " acte politique de désobéissance civile " (p. 294).

La première partie, historique, présente différents éléments des rapports entre États expliquant l'occupation américaine d'Haïti sous les angles sociologique, philosophique et économique. Le premier chapitre de philosophie politique relève les différences et les similitudes entre deux révolutions menées dans des colonies pour " rompre avec par et pour la métropole » (p. 24). Le deuxième chapitre revient sur le droit et le devoir d'ingérence que s'octroient les États les plus stables économiquement pour éviter la précarité d’autres États de la même aire géographique, au nom de valeurs telles que la démocratie ou le progrès, et il évoque de manière historique 
différentes étapes annonçant l'occupation américaine. Le troisième chapitre est davantage tourné vers les enjeux économiques au sein du Nouveau-Monde : il décrit les relations entre des États caraïbéens (Cuba, Haïti, République dominicaine) et l'État américain. À partir d'ouvrages d'époque, le quatrième chapitre fournit des éléments contextuels des relations entretenues avec Haïti, des points de vue américain (État dominant Haïti pendant l'occupation) et dominicain (région dominée par Haïti de 1822 à 1844, avant son indépendance).

La deuxième partie de l'ouvrage évoque les effets de l'occupation au travers d'analyses de pratiques de résistances mises en œuvre par des intellectuels (écrivains, journalistes et politiciens) de l'époque. Deux chapitres analysent des œuvres littéraires rédigées sur le mode fictionnel engagé (Le Choc et Le Joug) critiquant l'occupation américaine. Les deux autres chapitres évoquent le parcours de Jacques Roumain, auteur d'une autre œuvre engagée, Gouverneur de la Rosée, et homme politique de gauche.

La troisième partie fournit des analyses compréhensives de manifestations de résistance ou de pratiques d'insoumission plus populaires que dans la section précédente. Populaire est à entendre ici au sens d'éléments répandus parmi l'ensemble du peuple, quelle que soit la classe sociale, qu'il s'agisse de la poursuite de pratiques religieuses décriées par l'occupant (chapitre 9), de la création d'une langue dans certaines zones géographiques pour ne pas être compris de l'occupant (chapitre 11) ou de la transmission sur un mode mythique du passé de résistance armée de l'oppression coloniale française à l'occupation américaine au travers d'œuvres picturales très connues des Haïtiens (chapitre 12). Le chapitre 10, sur l'éducation comme lieu de résistance, semble moins relever de cette partie consacrée à l'insoumission populaire : il est davantage tourné vers les résistances politiques des Haïtiens à la mise en œuvre d'un nouveau système éducatif par les Américains que vers les pratiques de scolarisation des familles.

\section{Organisation du volume}

Les approches théoriques mobilisées dans cet ouvrage concernant la culture et l'identité ne relèvent pas d'une approche sociohistorique (Misantrope et Costalat-Founeau, 2014) ou sociocognitive (Berteaux, 2010), ni d'une approche clinique (Moussa-Babaci, 2016) : c'est un cadre d'analyse davantage tourné vers les institutions et l'action politique mené par certains individus qui est mobilisé par les auteurs. Ce positionnement en sciences sociales, partagé par les différents contributeurs qu'ils soient en sociologie ou en littérature par exemple, est enrichi d'un travail d'élaboration collective du phénomène étudié. Loin de constituer une collection de contributions individuelles, l'ouvrage, grâce surtout à l'introduction et la conclusion générales ainsi qu'au premier chapitre, offre une investigation commune du phénomène d'insoumission en tant que résistance à l'oppression résultant d'une relation coloniale, économique ou idéologique.

Cet important travail d'érudition en sciences sociales portant sur une période historique récente peut intéresser un large public en raison du style clair et accessible de tous les chapitres. Si l'étude linguistique du parler bolith peut sembler ardu, il faut noter son caractère pédagogique, qui offre la possibilité à un chercheur non spécialisé en linguistique de comprendre les enjeux linguistiques et identitaires qui lui sont liés. La présence du résumé de chaque contribution en début de chapitre facilite la navigation au sein de l'ouvrage. Mentionnons également la présentation en entonnoir des différentes parties qui, au delà du choix éditorial, offre une dimension didactique : les chapitres semblent se répondre, les premiers fournissant les éléments contextuels nécessaires à la compréhension des suivants, plus tournés vers des formes particulières de résistance. La démarche scientifique est perceptible grâce à la présentation dans chaque chapitre des sources correspondantes, avec une présentation spécifique à chaque contribution. Ce format est intéressant pour sensibiliser des étudiants à la démarche d'investigation historique. Les professionnels œuvrant dans l'humanitaire en Haïti ou travaillant avec la diaspora peuvent également consulter cet ouvrage avec profit. En effet, connaître les mouvements sociaux et politiques passés et leurs répercussions dans l'imaginaire collectif haïtien contribue à une meilleure compréhension de certains éléments présents aujourd'hui dans les institutions haïtiennes ou plus généralement dans les vécus et les relations au sein des diasporas caraïbéennes.

Alterstice - Revue Internationale de la Recherche Interculturelle, vol. 8, $n^{\circ} 1$ 


\section{Commentaires}

Au niveau formel, quelques coquilles subsistent, mais n'empêchent nullement la compréhension des propos. Par ailleurs, certaines contributions semblent moins abouties que d'autres, notamment le chapitre 10 sur la résistance dans le domaine de l'éducation, ou le chapitre 4 sur les usages des désignations d'occupant et d'occupé. Le statut de ce dernier chapitre, qualifié par son auteur de note de lecture, invite le lecteur à se poser la question de sa pertinence pour le propos général. Malgré tout, il contribue à penser de manière plus générale les relations néocoloniales et intracoloniales dans la Caraïbe.

La centration exclusive sur la situation haïtienne pendant l'occupation mise en perspective avec la question des colonisations et des asservissements pourrait faire penser à un ouvrage relevant du courant des postcolonial studies. L'utilisation, dans différentes contributions, de termes renvoyant à la couleur de peau et à l'ethnicisation des rapports à l'époque évoque, quant à elle, celui des black studies : " premier pays nègre des Amériques » (p. 7), « pour les fondateurs de la République d’Haïti la liberté devait franchir les limites épidermiques qu'elle connaissait jusqu'ici » (p. 21), " l'indigénisme [pour Jacques Roumain] avance que les Haïtiens sont des Nègres, d'origine africaine » (p. 182). Cet ouvrage s'inscrit-il dans l'une ou l'autre de ces approches ? Les éléments historiques fournis dans les différentes contributions de l'ouvrage permettent certes de prendre conscience de l'importance de mener des travaux en sciences humaines et sociales dans la Caraïbe ou de montrer l'apport de la révolution haïtienne aux définitions des droits de l'Homme et de la liberté. Cependant, les auteurs ne se réfèrent pas au courant des black studies ou à celui des postcolonial studies. Les responsables de l'ouvrage espèrent plutôt que l'analyse du cas haïtien puisse être un catalyseur pour étudier les relations internationales de l'époque de manière générale, et non du point de vue des relations coloniales ou héritées de la période de l'esclavage. De même, comme l’indique la présentation de l'ouvrage par l'éditeur, le cas haïtien peut se révéler utile pour interroger autrement l'actualité mondiale contemporaine, non du point de vue des grandes puissances économiques mais avec d'autres points de vue, dont celui d'une des premières républiques contemporaines.

La présente recension est probablement liée à mon orientation en psychologie de l'éducation. D'autres lecteurs issus d'autres horizons pourraient aborder autrement les propositions conceptuelles renvoyant à la question des droits collectifs ou les résultats d'analyses concernant la non-reconnaissance de la révolution haïtienne en tant qu'élément de modernité. II me semble manquer à cet ouvrage l'approche théorique interculturelle, qui prend en compte les situations de rencontre de cultures (Clanet, 1990; Berry, Poortinga, Segall et Dasen, 2002). Elle permettrait de penser les interrelations entre les porteurs de cultures différentes de l'époque et elle pourrait servir de garde-fou pour le chercheur dans son travail de décentration sociohistorique. De plus, étant donné le statut de société créole d'Haïti (Benoist, 2012), la démarche interculturelle, qui s'intéresse à l'asymétrie dans les rencontres comme celle originelle présente dans les espaces métissés caraïbéens, fournirait un cadre d'investigation pour comprendre et expliquer l'organisation des éléments universels et singuliers dans la mémoire collective concernant la notion de résistance et les comportements culturellement acceptables d'insoumission. Ceci étant dit, cet ouvrage constitue une contribution pluridisciplinaire importante aux débats toujours d'actualité entre le devoir d'ingérence entre États et le devoir collectif ou individuel d'insoumission. Sa lecture permet, au travers de l'analyse de la situation haïtienne, de relever des éléments universels concernant la résistance à l'oppression et d'autres plus singuliers, renvoyant par exemple à la complexité de la reconnaissance du statut d’État libre, indépendant et démocratique d'anciennes colonies, ce qui a un impact sur les formes d'ingérence et d'oppression générées. Ce travail inédit, en langue française, confirme l'intérêt de décentrer les regards, y compris celui des scientifiques, pour étudier les phénomènes sociaux.

\section{Références bibliographiques}

Benoist, J. (2012). La créolisation : locale ou mondiale ?, Archipélie, 3-4, 12-30.

Berry, J. W., Poortinga, Y. H., Segall, M. H. et Dasen, P. R. (2002). Cross-cultural psychology: research and applications ( 2 éd. revue). Cambridge, England : Cambridge University Press.

Berteaux, P. (2010). Influence des modes d'enculturation scolaire sur les styles cognitifs : I'exemple des écoles coranique et laïque à La Réunion et aux Comores, Carrefours de l'éducation, 29, 215-238. 
Clanet, C. (1990). L'interculturel. Introduction aux approches interculturelles en éducation et en sciences humaines. Toulouse : Presses universitaires du Mirail.

Misantrope, Y. et Costalat-Founeau, A. -M. (2014). Identité nationale ou identité régionale ? Une étude de cas à travers le conflit martiniquais de février 2009, Alterstice, 4(1), 45-60.

Moussa-Babaci, F. (dir.). (2016). Pratiques culturelles, contextes de violence et identité. Sarrebruck (Allemagne) : Éditions universitaires européennes.

Thésée, G. et Carr, P. R. (2014). La (re)lecture des mots, du monde et des maux des jeunes noirs : apports de la pédagogie critique à la recherche en éducation en contextes de racialisation, Revue canadienne de l'éducation, 37(1), 308-329. 